\title{
Silencing HCCR2 expression inhibits the proliferation of leukemia cells by inducing apoptosis and promoting cell cycle arrest
}

\author{
SHU-KAI QIAO, HAN-YUN REN, YONG-JIN SHI and WEI LIU \\ Department of Hematology, Peking University First Hospital, Beijing 100034, P.R. China
}

Received July 8, 2013; Accepted September 30, 2013

DOI: $10.3892 / \mathrm{ijmm} .2013 .1518$

\begin{abstract}
The human cervical cancer oncogene (HCCR2) has been found to be overexpressed in a variety of human malignant tumors cells, and its function is related to cell cycle progression and survival. However, the molecular mechanisms of action of HCCR2 in leukemia remain unclear. In this study, we used the RNA interference strategy to investigate the effects of HCCR2 knockdown in the K562 leukemia cell line, and to explore the potential mechanisms involved. Following transfection with small interfering RNA (siRNA) targeting HCCR2 (HCCR2-siRNA), we examined the effects of HCCR2 knockdown on cell morphology, cell proliferation, cell cycle progression and apoptosis in K562 cells. Morphological changes were evaluated by Wright-Giemsa staining. Cell cycle progression and apoptosis were measured by flow cytometry. The expression levels of genes related to the cell cycle and apoptosis were detected by quantitative RT-PCR (qRT-PCR) and western blot analysis. HCCR2 expression at the mRNA and protein level was significantly decreased following transfection with plasmids expressing HCCR2-siRNA. Silencing HCCR 2 expression significantly suppressed cell proliferation, induced G1 cell cycle arrest and promoted the apoptosis of K562 cells. Additionally, we found that the expression of Bax, p53 and p21 was significantly increased, while Bcl-2 expression was significantly decreased in the HCCR2-siRNA-transfected cells. However, the expression of p27 was not affected. These results suggest that the HCCR2 gene plays an important role in the tumorigenesis of leukemia, thus making it an attractive therapeutic target for acute leukemia.
\end{abstract}

\section{Introduction}

Acute leukemia is the most common hematological malignancy that is characterized by an uncontrolled expansion of

Correspondence to: Professor Han-Yun Ren, Department of Hematology, Peking University First Hospital, 8 Xishiku Street, Xicheng, Beijing 100034, P.R. China

E-mail: renhy0813@163.com

Key words: human cervical cancer oncogene, small interfering RNA, apoptosis, cell cycle, acute leukemia clonal leukemia cells in the bone marrow and/or peripheral blood often accompanied with anemia, bleeding and frequent infectious complications (1-4). Acute leukemia is generally classified as either acute myelogenous leukemia (AML) or acute lymphoblastic leukemia (ALL) and the primary treatments are remission induction therapy and post-remission chemotherapy $(1,2)$. In recent years, with the introduction of high-dose chemotherapy followed by allogeneic hematopoietic stem cell transplantation (allo-HSCT) and the discovery of novel therapeutic agents, considerable progress has been made in the outcomes of patients with acute leukemia (3-5). However, acute leukemia currently remains an incurable malignancy due to the complex molecular mechanisms behind its tumorigenesis $(6,7)$. Thus, the identification of novel molecular targets is mandatory for the early diagnosis and long-term management of acute leukemia.

The human cervical cancer oncogene (HCCR) was first identified from human cervical tissue through the differential display RT-PCR method by Ko et al (8). The HCCR gene maps to the long arm of the chromosome 12 and is classified into two types, HCCR1 and HCCR2 according to their molecular characteristics. A comparison of both sequences has revealed that HCCR 2 lacks the exon 1 of HCCR1 $(8,9)$. Previous studies have demonstrated that HCCR2 is not only overexpressed in human cervical cancer tissues, but is expressed at high levels in a variety of human malignancies, including breast, kidney, stomach, colon, liver and ovarian cancer (8-13). The functional role of HCCR 2 in tumorigenesis may involve the negative regulation of the p53 tumor suppressor gene $(8,13,14)$. Animal experiments have demonstrated that HCCR2-transgenic nude mice form spontaneous breast tumors, which further confirms the crucial role of HCCR2 in tumorigenesis (10). In our previous study, we observed that HCCR 2 mRNA was overexpressed in patients with acute leukemia and leukemia cell lines, such as K562 and HL-60 cells, but not in normal leukocytes (15). Therefore, HCCR 2 shows great promise as an important molecular target for the diagnosis and treatment of acute leukemia. Although accumulating data have shown that HCCR2 expression in many solid tumors correlates with clinical outcome has been confirmed as a good biomarker for monitoring disease progression, its specific mechanisms of action in acute leukemia remain elusive (10-13).

In the present study, we examined whether silencing HCCR2 expression using small interfering RNA (siRNA) exerts significant anti-proliferative effects in K562 cells. Furthermore, we 
also wished to investigate the potential molecular mechanisms behind these effects, which may help identify HCCR 2 as a novel therapeutic target for acute leukemia.

\section{Materials and methods}

Cell line and cell culture. The K562 cell line, established from human chronic myelogenous leukemia (CML) cells in blastic crisis, was purchased from the Cell Culture Center in Chinese Academy of Medical Sciences (Shanghai, China). The cells were cultured in RPMI-1640 medium (Invitrogen Life Technologies, Carlsbad, CA, USA) supplemented with $10 \%$ fetal bovine serum (HyClone, Logan, UT, USA), $100 \mu \mathrm{g} / \mathrm{ml}$ penicillin, $10 \mu \mathrm{g} / \mathrm{ml}$ streptomycin and $2 \mathrm{mmol} / \mathrm{l} \mathrm{L}$-glutamine. The cells were maintained in $\log$ phase growth at $37^{\circ} \mathrm{C}$ in a humidified atmosphere containing $5 \% \mathrm{CO}_{2}$.

Silencing HCCR 2 expression by siRNAs. We used the K562 cells to perform the HCCR2 silencing experiment, as a high expression level of HCCR2 in K562 cells was demonstrated in our previous study (15). The siRNAs used for the silencing of HCCR2 were designed and synthesized from Invitrogen Life Technologies according to previous literature (13). The oligonucleotide sequences used to generate the three synthesized HCCR2-targeting siRNA are listed in Table I. siRNA-H1, targeting HCCR2 mRNA coding sequence 475-493 bp; siRNA-H2, targeting HCCR2 mRNA coding sequence 611-629 bp; and siRNA-H3, targeting HCCR2 mRNA coding sequence $854-872$ bp. A scrambled sequence ACTACCGTTGTATAGGTGT was used as the negative control siRNA that is absent in human, mouse, and rat genomes. Each oligonucleotide pair was annealed by incubation at $95^{\circ} \mathrm{C}$ for $5 \mathrm{~min}$ and cooled slowly, and was ligated separately into plasmid vector pcDNA3 (Invitrogen Life Technologies), which had been digested with BamHI and EcoRI. These HCCR2siRNA vectors were transfected into the K562 cells using Lipofectamine 2000 (Invitrogen Life Technologies) according to the manufacturer's instructions. The ability of the three transfected HCCR2-siRNAs to silence HCCR2 expression was investigated by quantitative RT-PCR (qRT-PCR) and western blot analysis.

Cell proliferation assay. Cell proliferation was determined in cells in 96-well plates using the 3-(4,5-dimethylthiazol-2-yl)5-(3-carboxymethoxyphenyl)-2-(4-sulfophenyl)-2H-tetrazolium (MTS)/phenazine ethosulfate (PES) assay (Promega Corp., Madison, WI, USA) according to manufacturer's instructions. In brief, the blank control cells, and the negative control- and HCCR2-siRNA-transfected K562 cells were seeded in 96-well plates at densities of $5 \times 10^{3}$ cells/well. Cultures were set up in triplicate and maintained at $37^{\circ} \mathrm{C}$ in a humidified atmosphere with $5 \% \mathrm{CO}_{2}$. At $24,48,72,96$ and $120 \mathrm{~h}$ after transfection, $10 \mu \mathrm{l}$ MTS-PES (10 mg/ml) (Promega Corp.) were added into each well for an additional $6 \mathrm{~h}$ of incubation. A microplate reader was then used to measure the absorbance value at $490 \mathrm{~nm}$ for each well, which represented K562 cell proliferation.

Morphological observation of K562 cells. After transfection with HCCR2-siRNA, the K562 cells were smeared on microscope glass plates and stained with Wright-Giemsa. The morphological changes in the K562 cells was observed under a light microscope.

Cell cycle analysis and assessment of apoptosis. Cell cycle distribution was determined using DNA staining with propidium iodide (PI) (BioLegend, San Diego, CA, USA) followed by flow cytometry. Apoptosis assay was performed according to the directions contained in the manual provided with the Annexin V/PI apoptosis assay kit (BioLegend). In brief, the cells were harvested and washed twice with cold phosphatebuffered saline (PBS), and then the cells were resuspended in $1 \mathrm{X}$ binding buffer at a concentration of $1 \times 10^{6}$ cells $/ \mathrm{ml}$. A total of $100 \mu \mathrm{l}$ of the solution was then transferred to a $5-\mathrm{ml}$ culture tube with $5 \mu \mathrm{l}$ of Annexin V-FITC and $10 \mu \mathrm{l}$ of PI $(20 \mathrm{mg} / \mathrm{ml})$. The cells were gently vortexed and incubated for $15 \mathrm{~min}$ at room temperature in the dark. After the addition of $400 \mathrm{ml}$ of $1 \mathrm{X}$ binding buffer, the cells were analyzed by flow cytometry. Annexin $\mathrm{V}^{-}$and $\mathrm{PI}^{-}$cells were considered as viable cells. Annexin $\mathrm{V}^{+}$and $\mathrm{PI}^{-}$cells were considered as early-stage apoptotic cells. Annexin $\mathrm{V}^{+}$and $\mathrm{PI}^{+}$cells were considered as late-stage apoptotic cells.

qRT-PCR. Total RNA was isolated from the K562 cells using the TRIzol reagent (Invitrogen Life Technologies) according to the manufacturer's instructions, and then cDNA was synthesized from $2 \mu \mathrm{g}$ total RNA using a first-strand cDNA synthesis kit (Invitrogen Life Technologies). The PCR amplification protocol was as follows: denaturation at $94^{\circ} \mathrm{C}$ for $10 \mathrm{~min}$, 40 cycles of $94^{\circ} \mathrm{C}$ for $15 \mathrm{sec}, 58^{\circ} \mathrm{C} 30 \mathrm{sec}$, and $72^{\circ} \mathrm{C}$ for $40 \mathrm{sec}$. The mRNA expression levels of HCCR2, Bcl-2, Bax, p21, p27 and $\beta$-actin were detected using ABI 7900 (Applied Biosystems, Foster City, CA, USA) and SYBR-Green chemistry. The relative quantification of the target genes was calculated using the $2^{-\Delta \Delta \mathrm{Ct}}$ formula by the comparative cycle threshold $(\mathrm{Ct})$ value method (16). The PCR primers were designed based on the corresponding gene structure, and the sequences are listed in Table II. qRT-PCR assays were performed in triplicate.

Western blot analysis. The K562 cells were harvested and subsequently protein was separated by $15 \%$ SDSpolyacrylamide gel electrophoresis (SDS-PAGE) before being transferred onto a polyvinylidene difluoride (PVDF) membrane. The blots were blocked with 5\% non-fat dry milk in Tris-buffered saline containing $0.1 \%$ Tween-20 (TBST) at $37^{\circ} \mathrm{C}$ for $2 \mathrm{~h}$, followed by incubation with specific primary antibodies against HCCR2, Bcl-2, Bax, p21, p27 or $\beta$-actin (Santa Cruz Biotechnology Inc., Santa Cruz, CA, USA) at $4^{\circ} \mathrm{C}$ overnight. The blots were washed with TBST and incubated with a horseradish peroxidase-conjugated secondary antibody at $37^{\circ} \mathrm{C}$ for $2 \mathrm{~h}$. After several washes with TBST, the immunoreactive bands were visualized on X-ray film and scanned. All experiments were performed in triplicate. The quantitative data from bands were expressed as the target protein $/ \beta$-actin ratio using BandScan analysis software.

Statistical analysis. All statistical data are presented as the means \pm standard deviation (SD). The effects of treatment among the different groups were compared using a one-way analysis of variance. All analyses were performed using GraphPad Prism software version 5.0 (GraphPad Software Inc., San Diego, CA, 
Table I. Sequences used to generate the three synthesized HCCR2-targeting siRNA.

\begin{tabular}{lc}
\hline No. & Primer sequences \\
\hline siRNA-H1 & 5'-GATCCGACAGATCTGTGCACCAAGATCAAGACGTCTTGGTGCACAGATCTGTTTTTTTA-3' \\
siRNA-H2 & 3'-GCTGTCTAGACACGTGGTTCTAGTTCTGCAGAACCACGTGTCTAGACAAAAAAATTCGA-5' \\
& 5'-GATCCGTAAGATGTGAGAAGCATGGTCAAGACGCCATGCTTCTCACATCTTATTTTTTA-3' \\
siRNA-H3 & 3'-GCATTCTACACTCTTCGTACCAGTTCTGCGGTACGAAGAGTGTAGAATAAAAAATTCGA-5' \\
& 5'-GATCCGTTGTGCAGCAAGAGAGACATCAAGACGTGTCTCTCTTGCTGCACAATTTTTA-3' \\
& 3'-GCAACACGTCGTTCTCTCTGTAGTTCTGCACAGAGAGAACGACGTGTTAAAAAATCGA-5'
\end{tabular}

HCCR2, human cervical cancer oncogene 2; siRNA, small interfering RNA.

Table II. Primers used for quantitative RT-PCR.

\begin{tabular}{|c|c|c|}
\hline Gene & & Primer sequences \\
\hline \multirow[t]{2}{*}{ HCCR2 } & Sense & 5'-GGAGGCAGAGAGAGGAGCAG-3' \\
\hline & Antisense & 5'-AGCAAGAGGGTTTGTTTCAGTTCT-3' \\
\hline \multirow[t]{2}{*}{$\mathrm{Bcl}-2$} & Sense & 5'- ATCGCCCTG TGGATGACTGAG-3' \\
\hline & Antisense & 5'-CAGCCAGGAGAAATCAAACAGAGG-3 \\
\hline \multirow[t]{2}{*}{ Bax } & Sense & 5'-GGACGAACTGGACAGTAACATGG-3' \\
\hline & Antisense & 5'-GCAAAGTAGAAAAGGGCGACAAC-3' \\
\hline \multirow[t]{2}{*}{ p21 } & Sense & 5'-GCAGACCAGCATGACAGATTT-3' \\
\hline & Antisense & 5'-GGATTAGGGCTTCCTCTTGGA-3' \\
\hline \multirow[t]{2}{*}{ p27 } & Sense & 5'-CCTCTTCGGCCCGGTGGAC-3' \\
\hline & Antisense & 5'-TTTGGGGAACCGTCTGAAAC-3' \\
\hline \multirow[t]{2}{*}{ p53 } & Sense & 5'-TGCTCAGATAGCGATGGTC-3' \\
\hline & Antisense & 5'-TAGGGCACCACCACACTAT-3' \\
\hline \multirow[t]{2}{*}{$\beta$-actin } & Sense & 5'-TCTGGCACCACACCTTCTACAATG-3' \\
\hline & Antisense & 5'-AGCACAGCCTGGATAGCAACG-3' \\
\hline
\end{tabular}

USA) and a value of $\mathrm{P}<0.05$ was considered to indicate a statistically significant difference.

\section{Results}

Inhibition of HCCR2 expression by siRNAs in K562 cells. We analyzed the ability of the three HCCR2-siRNAs to suppress HCCR 2 mRNA expression by qRT-PCR. The results revealed that transfection with the three HCCR2-siRNAs resulted in varying degrees of reduced HCCR 2 mRNA expression in the K562 cells. The quantification analysis revealed that siRNA-H1 $(\mathrm{P}<0.01)$, siRNA-H2 $(\mathrm{P}<0.05)$ and siRNA-H3 $(\mathrm{P}<0.01)$ significantly inhibited HCCR2 expression at the mRNA level compared with the negative control cells (Fig. 1A). The western blot analysis results also indicated that HCCR 2 protein expression was significantly suppressed following transfection with the three HCCR2-siRNAs (Fig. 1B). The HCCR2 mRNA and protein levels were not altered in the blank group cells or in the negative control siRNA-transfected cells. As siRNA-H3 showed the highest efficiency in silencing HCCR 2 expression, we thus selected siRNA-H3 for the subsequent experiments.

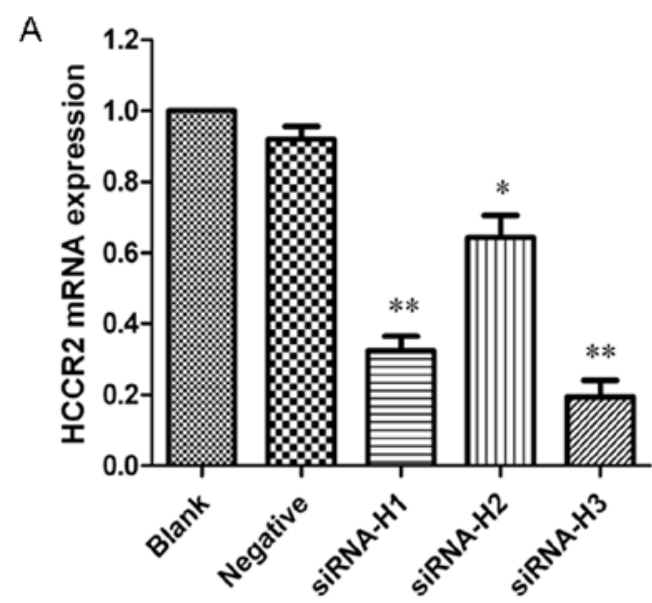

B

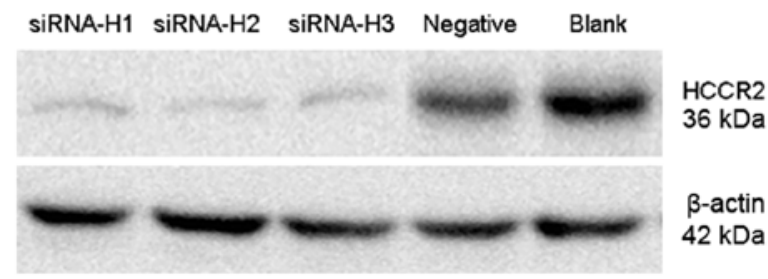

Figure 1. Effects of transfection with small interfering RNA (siRNA) targeting the human cervical cancer oncogene (HCCR2). (A) HCCR2 mRNA expression in the blank control, and negative control- and the three HCCR2-siRNA-transfected cells was detected by quantitative RT-PCR after transfection. (B) HCCR2 protein expression was measured by western blot analysis after transfection with HCCR2-siRNA. The upper lane indicates that the HCCR 2 protein expression was markedly inhibited in the HCCR2-siRNAtransfected cells compared with the blank control cells. Data represent the means \pm standard deviation $(\mathrm{SD})$ of three independent experiments. ${ }^{*} \mathrm{P}<0.05$, ${ }^{* *} \mathrm{P}<0.01$ compared with the the blank control or negative control cells.

HCCR2 knockdown suppresses the proliferation of $K 562$ cells. Our results revealed that silencing the HCCR2 gene affected the proliferation of K562 cells. As shown in Fig. 2, cell proliferation was markedly inhibited in the HCCR2-siRNAtransfected cells from day 4 to 6 after transfection when compared with the blank control and the negative control cells $(\mathrm{P}<0.01)$. However, there was no statistically significant difference in cell proliferation during the first three days among the three groups of cells. 


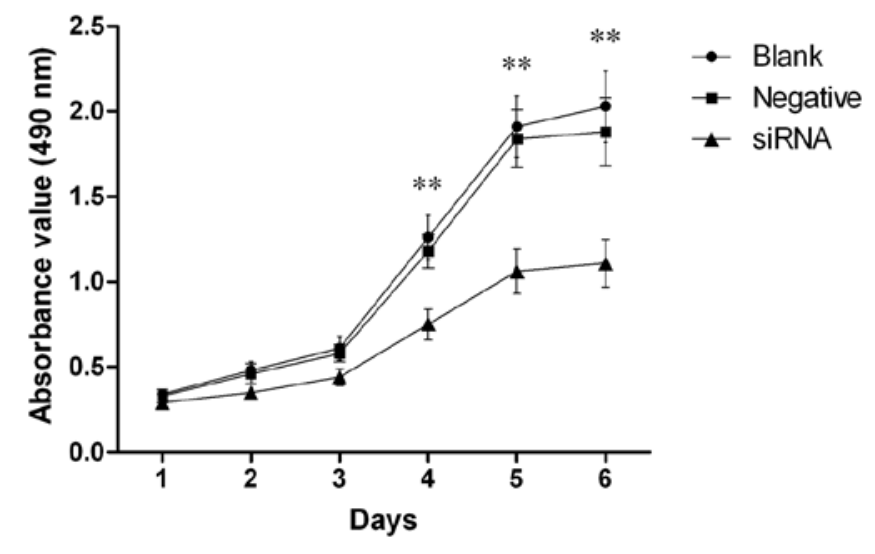

Figure 2. Transfection with small interfering RNA (siRNA) targeting human cervical cancer oncogene (HCCR2) inhibits the proliferation of K562 cells. The blank control, and negative control- and HCCR2-siRNA-transfected K562 cells were seeded in 96-well plates at initial densities of $5 \times 10^{3}$ cells/ well, and then analyzed by MTS/PES assay with the measurement of absorbance at $490 \mathrm{~nm}$. Data represent the means \pm standard deviation (SD) of three independent experiments. ${ }^{* *} \mathrm{P}<0.01$, compared with the blank control cells.

Morphological changes in K562 cells following transfection with HCCR2-siRNA. After transfection for $96 \mathrm{~h}$, a morphological examination of the HCCR2-siRNA-transfected K562 cells revealed the typical appearance of apoptotic cells, which included the appearance of condensed nuclear chromatin, nuclei shrinkage, nuclear fragmentation and the formation of apoptotic bodies. However, these morphological changes were not observed among the blank control and negative control cells (Fig. 3).

HCCR 2 knockdown inhibits cell cycle progression and induces apoptosis. To determine whether HCCR2 knockdown results in cell cycle changes, the cellular DNA content was measured by flow cytometry. The results revealed that the percentage of K562 cells in the G0/G1 phase was significantly increased when compared with the blank control $(\mathrm{P}<0.01)$. By contrast, the percentage of $\mathrm{K} 562$ cells in the $\mathrm{S}$ phase was significantly decreased as compared with the blank control $(\mathrm{P}<0.01)$. However, the number of K562 cells in the G2/M phase was relatively unaffected. These data demonstrated that HCCR2 knockdown by siRNA induced a significant arrest of cell cycle progression in the G1 phase, resulting in a decreased number of cells in the $\mathrm{S}$ phase with a corresponding accumulation of cells in the G0-G1 phase (Fig. 4A). Fig. 4B presents a representative example of apoptotic cells in the blank control, and the negative control- and HCCR2-siRNA-transfected group. The results revealed that the ratio of early-stage and late-stage apoptotic cells in the HCCR2-siRNA-transfected cells was significantly higher than that in the blank control and negative control cells $(\mathrm{P}<0.01)($ Fig. 4C).

Effect of HCCR2 knockdown on the expression of apoptosis- and cell cycle-related genes. To further investigate the molecular mechanisms behind the suppression of cell proliferation and G0/G1 cell cycle arrest induced by transfection with HCCR2-siRNA, we analyzed the mRNA and protein expression levels of Bcl-2, Bax, p21, p27 and p53 in the K562 cells at $96 \mathrm{~h}$ after transfection by qRT-PCR and western blot

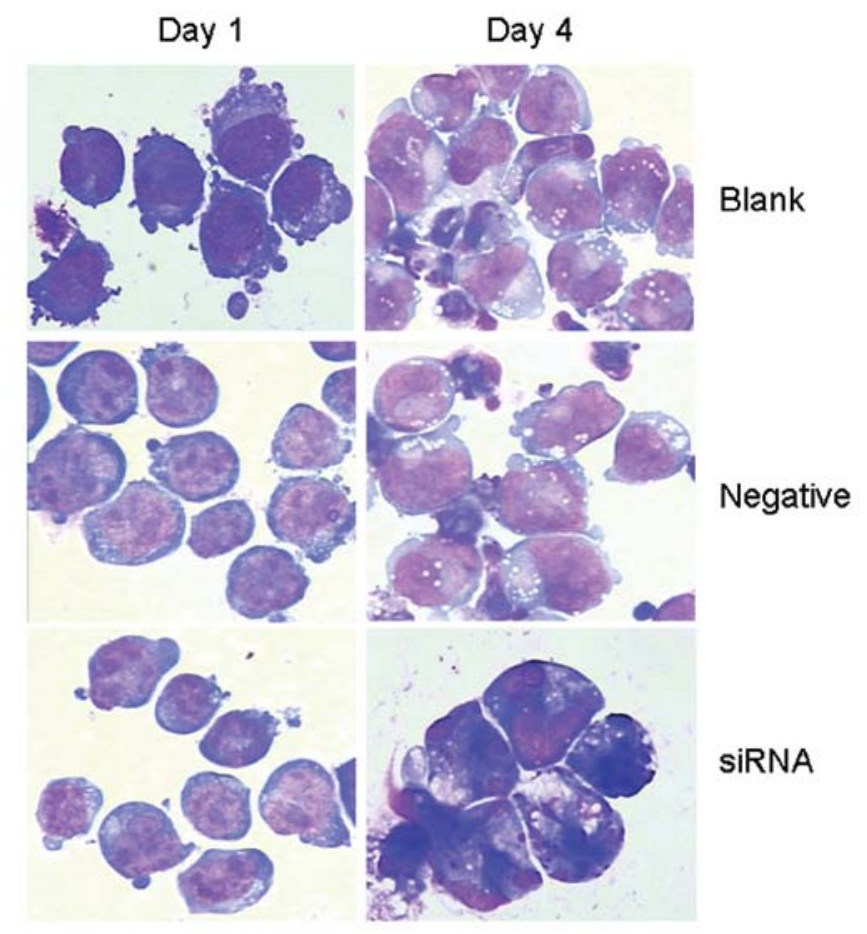

Figure 3. Morphological changes of K562 cells following transfection with small interfering RNA (siRNA) targeting human cervical cancer oncogene (HCCR2) (magnification, x1,000). The K562 cells were smeared on microscope glass plates and stained with Wright-Giemsa at $96 \mathrm{~h}$ after transfection. The typical appearance of apoptotic cells, which included the appearance of condensed nuclear chromatin, nuclei shrinkage, nuclear fragmentation and the formation of apoptotic bodies can be observed in the siRNA-transfected cells. Blank, untreated K562 cells; negative, K562 cells treated with scrambled nontargeting siRNA; siRNA, K562 cells treated with HCCR2-targeting siRNA.

analysis. Fig. 5A represents the relative mRNA quantification of these genes in the blank control group, and negative control- and HCCR2-siRNA-transfected K562 cells. The results indicated that $\mathrm{Bcl}-2$ mRNA expression significantly decreased $(\mathrm{P}<0.01)$, while Bax mRNA expression markedly increased $(\mathrm{P}<0.01)$ when compared with the blank control. Furthermore, the Bax/Bcl-2 ratio was also significantly increased in the HCCR2-siRNA-transfected cells. In addition, we also observed that the mRNA expression levels of p21 and p53 were markedly elevated in the HCCR2-siRNA-transfected K562 cells as compared with the blank control and negative control $(\mathrm{P}<0.01)$; however, 27 mRNA expression was not markedly altered in the three groups of cells. Representative immunoblots are shown in Fig. 5B. The western blot analysis results confirmed a marked decrease in Bcl-2 protein expression $(\mathrm{P}<0.01)$, and a significant increase in Bax, $\mathrm{p} 21$ and p53 protein expression following transfection with HCCR2-siRNA $(\mathrm{P}<0.01)$. No significant difference was observed in the $\mathrm{p} 27$ protein expression level among the three groups (Fig. 5C).

\section{Discussion}

siRNA is a powerful tool for gene knockdown, in which doublestranded RNA (dsRNA) is processed into siRNA, inducing the activation of cellular pathways, leading to selective sequencespecific silencing of target genes with homology to dsRNA (17). 

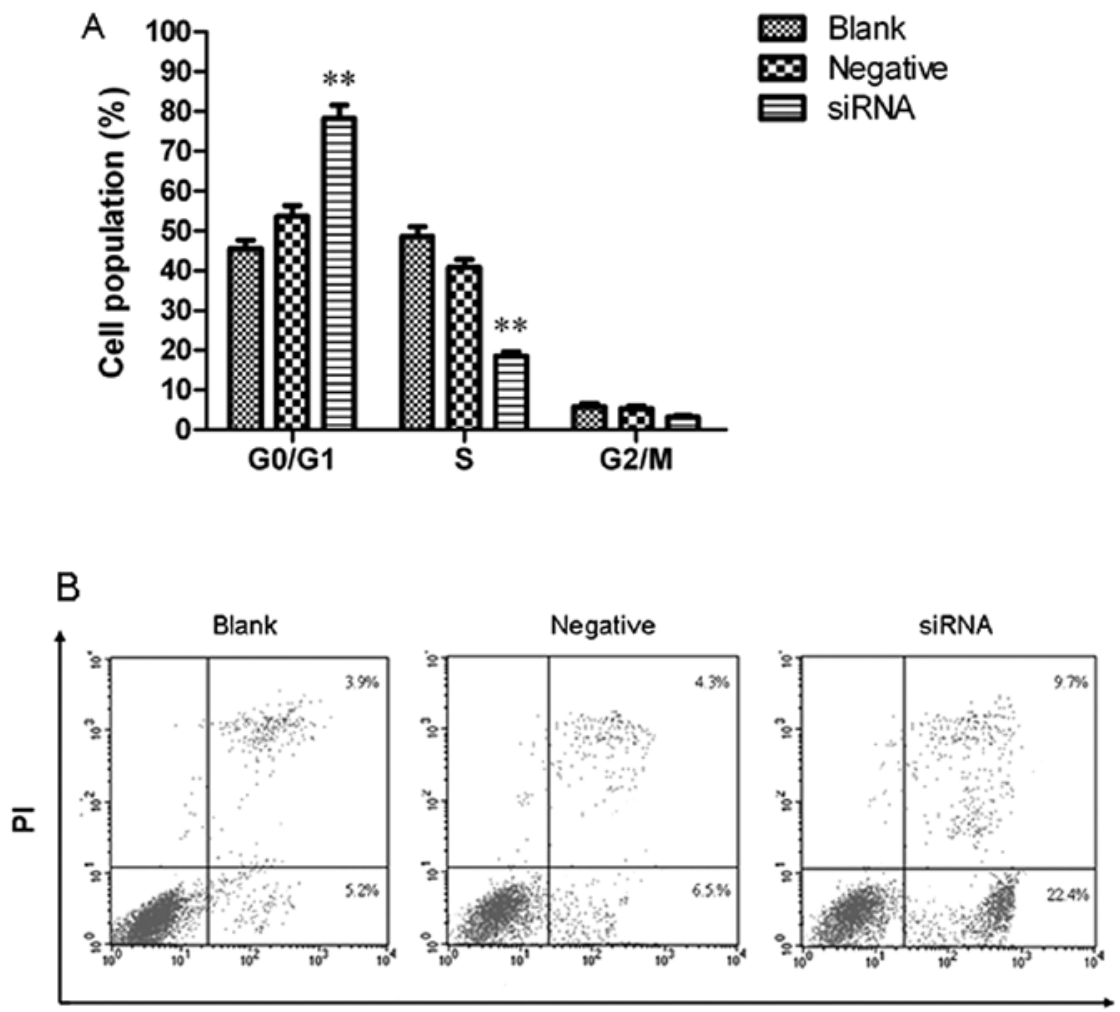

Annexin V

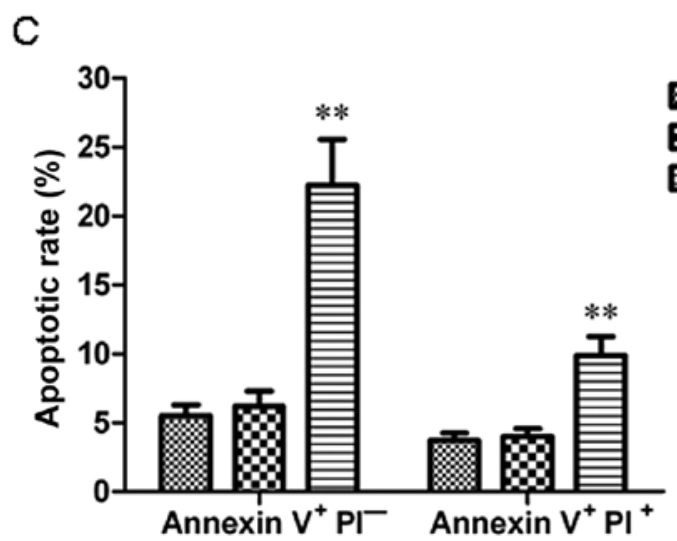

Figure 4. Silencing human cervical cancer oncogene (HCCR2) expression inhibits cell cycle progression and induces apoptosis. (A) The cell cycle progression was analyzed by flow cytometry. (B) Representative example of apoptotic cells after Annexin V/PI staining in the blank control, and negative control- and HCCR2-small interfering RNA (siRNA)-transfected K562 cells. (C) Apoptotic rate of K562 cells in the blank control, and negative control- and HCCR2-siRNAtransfected groups. Data represent the means \pm standard deviation (SD) of three independent experiments. ${ }^{* *} \mathrm{P}<0.01$, compared with the blank control cells.

A large number of studies have indicated that siRNA is an ideal method in molecular biology research and a very attractive therapeutic alternative to chemical drugs (17-19). In the present study, in order to investigate the function role of the HCCR2 gene in the tumorigenesis of acute leukemia, we silenced the expression of HCCR2 in K562 cells using siRNA. It is well known that K562 cells are derived from human CML cells in blastic crisis and are often used as an in vitro model of leukemia cells. Our results demonstrated that the mRNA and protein expression levels of HCCR2 were markedly decreased following transfection with HCCR2-targeting siRNA in K562 cells.

A previous report by Yoon et al (11) identified that HCCR2 was overexpressed in patients with hepatocellular carcinoma and thus may be recognized as a novel biomarker for hepa- tocarcinogenesis. The same study indicated that the HCCR2 assay has an advantage over the alpha-fetoprotein (AFP) assay in the early diagnosis of hepatocellular carcinoma. In our previous study, we found that the HCCR2 gene was abnormally overexpressed in newly diagnosed AML and ALL patients when compared with the normal control group. Furthermore, the patients who achieved complete remission after chemotherapy presented a significant decrease in HCCR2 expression; however, the patients with no remission showed not decrease in expression. In addition, the moment a patient relapsed, the level of HCCR 2 expression once again increased (15). These results indicate that HCCR2 may be used as biomarker for monitoring minimal residual disease in patients with acute leukemia. 


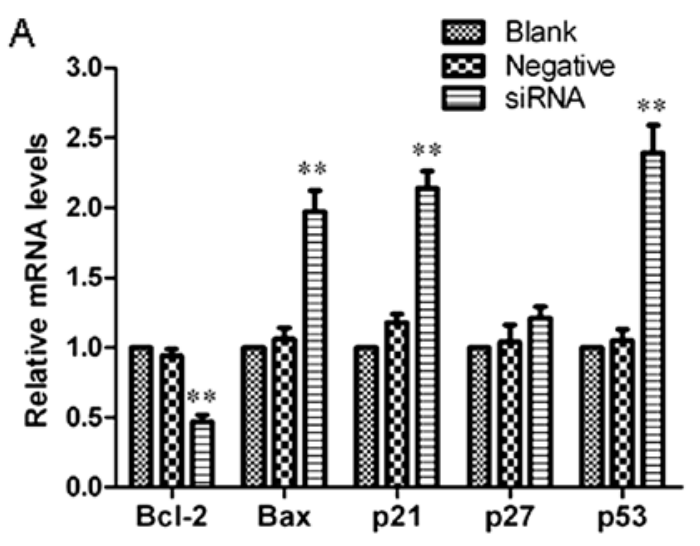

B
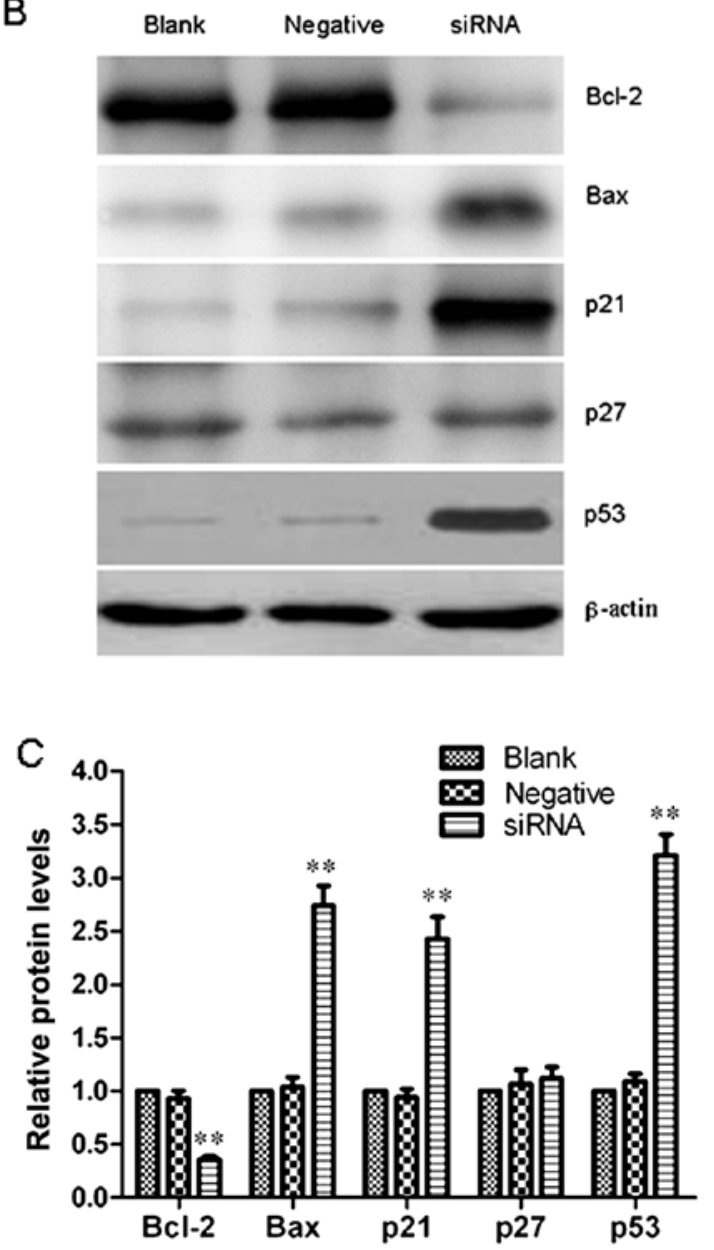

Figure 5. mRNA and protein expression of Bcl-2, Bax, p21, p27 and p53 in K562 cells following transfection with small interfering RNA (siRNA) targeting human cervical cancer oncogene (HCCR2). (A) The mRNA levels were measured at $96 \mathrm{~h}$ after transfection by quantitative RT-PCR. (B) Representative immunoblots of these proteins in the blank control, and negative control- and HCCR2-siRNA-transfected cells; the expression of $\beta$-actin was used as the loading control. (C) The protein levels were detected at $96 \mathrm{~h}$ after transfection by western blot analysis. Data represent the means \pm standard deviation (SD) of three independent experiments. ${ }^{* * *} \mathrm{P}<0.01$, compared with the blank control cells.

In this study, we examined whether silencing HCCR2 expression by siRNA affects cell proliferation, cell cycle progression and apoptosis in K562 cells. We observed that the cell growth of HCCR2-knockdown clones was significantly attenuated compared with the blank or negative control clones, while the cell numbers were comparable among all the groups at 4 days after transfection. Additionally, we measured the apoptosis of K562 cells using Annexin V/PI staining and found that the rate of early-stage and late-stage apoptotic cells was markedly increased in the HCCR2-siRNA-transfected cells. In the cell cycle analysis, a decreased number of cells in the $S$ phase and an increased number of cells in the G0/G1 phase were observed in the HCCR2-siRNA-transfected cells; however, the number of cells in the G2/M phase was relatively unaffected. Taken together, our results demonstrated that silencing HCCR2 expression effectively suppressed the proliferation and survival of K562 cells, inducing marked antitumor effects, which were associated with the induction of apoptosis and the inhibition of cell cycle G1/S phase transition.

p53 is a putative tumor suppressor gene, which plays a crucial role in controlling cell proliferation, and suppressing the growth and transformation of cells primarily through the induction of cell cycle arrest or apoptosis (20). The function of the p53 protein is to maintain the integrity of the genome and to repair damaged DNA. Before the initiation of damaged DNA repair, the p53 protein first induces the cells into G1 phase arrest. If DNA repair is not completed, p53 may induce the damaged cells into programmed cell death (21). In addition, the p53 protein is well known as the master regulator of the p21 gene $(22,23)$. In agreement with previous reports demonstrating the function of HCCR2 as a negative regulator of p53 in tumorigenesis $(8,13,14)$, we found that HCCR2 was overexpressed and accompanied with an almost null expression of p53 in K562 cells. Furthermore, p53 expression was markedly upregulated following transfection with HCCR2-siRNA, which was consistent with the increased number of apoptotic K562 cells. However, there have been conflicting reports, in which it was found that siRNA targeting HCCR2 did not alter the p53 protein expression level in HepG2 cells (24). This difference in the role of p53 may be explained by the fact that cells derived from various tissues may have different genetic backgrounds.

Cell cycle progression is regulated by cyclin-dependent kinases (CDKs), which are activated by cyclins binding or inhibited by CDK inhibitors (CKIs) (25). The p21 proteins belong to the first family of CKIs, which inhibit cell cycle progression mainly through the suppression of CDK2 activity and block the transition from the G1 phase into the $S$ phase after DNA damage (26-28). Although this activity is shared by other CKIs, such as p27 and p57, a number of clinical and experimental studies have suggested that p21 plays a crucial role in tumorigenesis (29-31). In this study, our data demonstrated that silencing HCCR 2 expression upregulated the expression of $\mathrm{p} 21$, but did not affect p27 expression in K562 cells.

Bcl-2, as an anti-apoptotic protein, inhibits apoptotic cell death and its overexpression is considered to promote survival in tumor cells (32). On the contrary, Bax is a pro-apoptotic protein, whose overexpression can inhibit the malignant progression of a tumor (33). The upregulation of the $\mathrm{Bax} / \mathrm{Bcl}-2$ ratio directs the cells more towards apoptosis than survival (34). In this study, we found that the knockdown of HCCR2 decreased Bcl-2 expression and increased Bax expression. Furthermore, the Bax/Bcl-2 ratio was significantly increased in the K562 cells following the knockdown of HCCR2 using siRNA. These results demonstrated that silencing HCCR2 expression induced 
a greater number of K562 cells into irreversible apoptosis by regulating the expression of $\mathrm{Bcl}-2$ and $\mathrm{Bax}$.

In conclusion, the results from the present study suggest that silencing HCCR2 gene expression by siRNA suppresses cell proliferation, promotes cell cycle G1 phase arrest and induces apoptosis in K562 cells. The data presented in this study also provide a mechanistic explanation for the antiproliferative and anti-apoptotic effects induced by silencing HCCR2 expression in K562 cells. Although the details of the functional mechanisms of HCCR2 in vivo require further investigation, HCCR2 may serve as an attractive molecular target for the treatment of leukemia.

\section{References}

1. Ferrara $\mathrm{F}$ and Schiffer CA: Acute myeloid leukaemia in adults Lancet 381: 484-495, 2013

2. Inaba H, Greaves M and Mullighan CG: Acute lymphoblastic leukaemia. Lancet 381: 1943-1955, 2013.

3. Hamilton BK and Copelan EA: Concise review: the role of hematopoietic stem cell transplantation in the treatment of acute myeloid leukemia. Stem Cells 30: 1581-1586, 2012.

4. Khaled SK, Thomas SH and Forman SJ: Allogeneic hematopoietic cell transplantation for acute lymphoblastic leukemia in adults. Curr Opin Oncol 24: 182-190, 2012.

5. Ungewickell A and Medeiros BC: Novel agents in acute myeloid leukemia. Int J Hematol 96: 178-185, 2012.

6. Roboz GJ: Current treatment of acute myeloid leukemia. Curr Opin Oncol 24: 711-719, 2012.

7. Kimby E, Nygren P and Glimelius B: A systematic overview of chemotherapy effects in acute myeloid leukaemia. Acta Oncol 40: 231-252, 2001

8. Ko J, Lee YH, Hwang SY, et al: Identification and differential expression of novel human cervical cancer oncogene HCCR-2 in human cancers and its involvement in p53 stabilization. Oncogene 22: 4679-4689, 2003.

9. Chung YJ and Kim JW: Novel oncogene HCCR: its diagnostic and therapeutic implications for cancer. Histol Histopathol 20 999-1003, 2005

10. Ko J, Shin SM, Oh YM, Lee YS, Ryoo ZY, Lee YH, Na DS and Kim JW: Transgenic mouse model for breast cancer: induction of breast cancer in novel oncogene HCCR-2 transgenic mice. Oncogene 23: 1950-1953, 2004.

11. Yoon SK, Lim NK, Ha SA, et al: The human cervical cancer oncogene protein is a biomarker for human hepatocellular carcinoma. Cancer Res 64: 5434-5441, 2004.

12. Jung SS, Park HS, Lee IJ, et al: The HCCR oncoprotein as a biomarker for human breast cancer. Clin Cancer Res 11: 7700-7708, 2005.

13. Ha SA, Lee YS, Shin SM, et al: Oncoprotein HCCR-1 expression in breast cancer is well correlated with known breast cancer prognostic factors including the HER 2 overexpression, p53 mutation, and ER/PR status. BMC Cancer 9: 51, 2009.

14. Ha SA, Shin SM, Lee YJ, et al: HCCRBP-1 directly interacting with HCCR-1 induces tumorigenesis through P53 stabilization. Int J Cancer 122: 501-508, 2008.

15. Qiao SK, Guo XN, Xu SR and Wang Y: The expression and clinical prognostic value of HCCR genes in patients with acute leukemia. Chin Journal Hematol 7: 481-483, 2010 (In Chinese).
16. Schmittgen TD and Livak KJ : Analyzing real-time PCR data by the comparative C (T) method. Nat Protoc 3: 1101-1108, 2008.

17. Ichim TE, Li M, Qian H, Popov IA, Rycerz K, Zheng X, White D, Zhong R and Min WP: RNA interference: a potent tool for genespecific therapeutics. Am J Transplant 4: 1227-1236, 2004.

18. Leung RK and Whittaker PA: RNA interference: from gene silencing to gene- specific therapeutics. Pharmacol Ther 107: 222-239, 2005.

19. Takeshita F and Ochiya T: Therapeutic potential of RNA interference against cancer. Cancer Sci 97: 689-696, 2006.

20. Olivier M, Petitjean A, Marcel V, Pétré A, Mounawar M, Plymoth A, de Fromentel CC and Hainaut P: Recent advances in p53 research: an interdisciplinary perspective. Cancer Gene Ther 16: 1-12, 2009.

21. Wang B, Xiao Z and Ren EC: Redefining the p53 response element. Proc Natl Acad Sci USA 106: 14373-14378, 2009.

22. Peller S and Rotter V: TP53 in hematological cancer: low incidence of mutations with significant clinical relevance. Hum Mutat 21: 277-284, 2003.

23. Janssen A, Schiffmann S, Birod K, Maier TJ, Wobst I, Geisslinger $\mathrm{G}$ and Grösch S: p53 is important for the anti-proliferative effect of ibuprofen in colon carcinoman cells. Biochem Biophys Res Commun 365: 698-703, 2008.

24. Guo J, Yang L, Zhang Y, Wang J, Wan S, Xia S, Yang S, Wang R and Fang D: Silencing of the HCCR2 gene induces apoptosis and suppresses the aggressive phenotype of hepatocellular carcinoma cells in culture. J Gastrointest Surg 15: 1807-1813, 2011.

25. Vermeulen K, Van Bockstaele DR and Berneman ZN: The cell cycle: a review of regulation, deregulation and therapeutic targets in cancer. Cell Prolif 36: 131-149, 2003.

26. Warfel NA and El-Deiry WS: p21WAF1 and tumourigenesis: 20 years after. Curr Opin Oncol 25: 52-58, 2013.

27. Stivala LA, Cazzalini O and Prosperi E: The cyclin-dependent kinase inhibitor p21CDKN1A as a target of anti-cancer drugs. Curr Cancer Drug Targets 12: 85-96, 2012.

28. Kizildag S, Ates H and Kizildag S: Treatment of K562 cells with 1,25-dihydroxyvitamin D3 induces distinct alterations in the expression of apoptosis-related genes BCL2, BAX, BCLXL, and p21. Ann Hematol 89: 1-7, 2010.

29. Cmielová J and Rezáčová M: p21Cip1/Waf1 protein and its function based on a subcellular localization [corrected]. J Cell Biochem 112: 3502-3506, 2011

30. Wang X, Gao P, Long M, Lin F, Wei JX, Ren JH, Yan L, He T, Han Y and Zhang HZ: Essential role of cell cycle regulatory genes p21 and p27 expression in inhibition of breast cancer cells by arsenic trioxide. Med Oncol 28: 1225-1254, 2011.

31. Coqueret O: New roles for $\mathrm{p} 21$ and $\mathrm{p} 27$ cell-cycle inhibitors: a function for each cell compartment? Trends Cell Biol 13: 65-70, 2003.

32. Tomek M, Akiyama T and Dass CR: Role of Bcl-2 in tumour cell survival and implications for pharmacotherapy. J Pharm Pharmacol 64: 1695-1702, 2012.

33. Yin C, Knudson CM, Korsmeyer SJ and Van Dyke T: Bax suppresses tumorigenesis and stimulates apoptosis in vivo. Nature 385: 637-640, 1997

34. Chiu TL and Su CC: Curcumin inhibits proliferation and migration by increasing the Bax to Bcl-2 ratio and decreasing NF- $\kappa$ Bp65 expression in breast cancer MDA-MB-231 cells. Int J Mol Med 23: 469-475, 2009. 